\title{
Management of Thrombotic Thrombocytopenic Purpura with Autoantibodies to ADAMTS-13 and Concurrent Preeclampsia in Pregnancy: Multidisciplinary Team Approach
}

\author{
Tiffany Patrick, M.D. ${ }^{1}$ Steve J. Carlan, M.D. ${ }^{1}$ Jose Eugenio Najera, M.D. ${ }^{2}$ Julie Eastwood, R.N. ${ }^{1}$ \\ ${ }^{1}$ Department of Obstetrics and Gynecology, Winnie Palmer Hospital, \\ Orlando Regional Healthcare, Orlando, Florida \\ 2 Department of Hematology/Oncology, MD Anderson Cancer Center \\ Address for correspondence and reprint requests Steve J. Carlan, \\ M.D., 105 West Miller Street, Orlando, FL 32806 \\ (e-mail: stevecarlan@gmail.com).
} Orlando, Orlando, Florida

Am J Perinatol Rep 2012;2:37-38.

\begin{abstract}
Keywords

- TTP

- severe preeclampsia

- multidisciplinary team

- autoantibodies

Background Thrombotic thrombocytopenic purpura (TTP) can present with many laboratory features of preeclampsia, which can make an accurate diagnosis difficult in late pregnancy. Because the treatments of TTP and preeclampsia are different and the clinical sequelae of delayed therapy potentially lethal, a rapid and accurate diagnosis is important.

Case Report We present a case of an acute episode of TTP secondary to acquired autoantibodies complicated by severe preeclampsia with headache and treated with corticosteroids, plasma exchange therapy, magnesium sulfate, and delivery. The postpartum course was complicated and resulted in a prolonged hospital stay. A multidisciplinary team was recruited as consultants.

Conclusion Concurrent TTP and severe preeclampsia can result in life-threatening complications. To ensure the best possible clinical outcome, an awareness of the medical systems' resources is required.
\end{abstract}

Thrombotic thrombocytopenic purpura (TTP) is a rare, severe life-threatening emergency characterized by microangiopathic hemolytic anemia, thrombocytopenia, acute renal insufficiency, altered mental status, and fever. It exists in both congenital and acquired forms and is associated with the absence or severe depletion of von Willebrand factor cleaving protease known as a disintegrin and metalloproteinase with thrombospondin-like repeats (ADAMTS-13). ${ }^{1,2}$ A deficiency in this protease can be congenital or the result of an acquired auto antibody to ADAMTS-13 and can lead to extensive platelet adhesion and clumping and possibly secondary end-organ damage. Plasma-based therapy containing replacements of ADAMTS- 13 was a major advancement for treatment of TTP. Today, high-dose intravenous methylprednisolone and plasma exchange are the mainstay treatments.

received

October 17, 2011

accepted after revision

December 1, 2011

published online

February 22, 2012

Pregnancy can precipitate the disease in first-time patients or can exacerbate its recurrence. However, if TTP occurs for the first time during pregnancy, it may mimic other severe conditions such as severe preeclampsia. Rather than plasma exchange, preeclampsia only responds to delivery of infant.

The incidence of TTP in pregnancy is 1 in $25,000^{3}$ compared with the 5 to $8 \%$ incidence of preeclampsia. ${ }^{4}$ Consequently, TTP may be easily overlooked by obstetric providers. Once diagnosed, however, all of the hospital's resources including maternal transport service, internal medicine service, critical care team, laboratory, blood bank, hospital educators, and neonatology and hematology services should be recruited.

We present a patient with TTP with inhibitors against ADAMTS-13 complicated by severe preeclampsia with

Copyright $\odot 2012$ by Thieme Medical Publishers, Inc., 333 Seventh Avenue, New York, NY 10001, USA. Tel: +1(212) 584-4662.
DOI http://dx.doi.org/ 10.1055/s-0032-1305799. ISSN 2157-6998. 
headache who was successfully treated with corticosteroids, plasma exchange therapy, delivery, and liberal use of the hospital resources.

\section{Case Report}

This was a 28-year-old G6, P1041 at 32.2 weeks who presented to the clinic for a routine prenatal visit and a known history of TTP secondary to ADAMTS-13 deficiency. The patient had been extensively educated by the staff about the potential effects of her disease on her pregnancy and was keeping a log of her weight and blood pressures along with a fetal movement chart. She had gained 20 pounds in 3 weeks, had a $6 / 10$ headache, $300 \mathrm{mg} / \mathrm{dL}$ protein, and blood pressure of $155 / 109$. All of her previous visits were characterized by normal urine dips and blood pressure, and home blood pressures had been normal. At this time, she was receiving plasmapheresis and $50 \mathrm{mg}$ of methylprednisolone three times weekly. Her platelet count had decreased from 100,000 to $76,000 / \mu \mathrm{L}$, and lactate dehydrogenase was 330 $\mathrm{IU} / \mathrm{L}$, an increase from $100 \mathrm{IU} / \mathrm{L}$. She was admitted with a diagnosis of severe preeclampsia with thrombocytopenia and hemolysis with possible acute TTP episode. Internal medicine and hematology oncology departments were consulted and the neonatal intensive care unit was notified. The laboratory, blood bank, and medical critical care team were called about the patient. Her liver enzymes returned as normal, fibrinogen was $439 \mathrm{mg} / \mathrm{dL}$, international normalized ratio was 0.9 , and reticulocyte count was $4.44 \%$. There was no laboratory evidence of renal involvement. Magnesium sulfate was started; she was given betamethasone for lung maturation, and neurology consultation was requested. No parenteral antihypertensive medication was required as her blood pressures fluctuated between 130/80 and 160/113. No abnormalities were found on the fetal ultrasound, and fetal surveillance was normal. Over the next 24 hours, her headache worsened and a multispecialty conference with the medical team, neonatology, maternal-fetal medicine, anesthesia, and nursing departments was held. The consensus was that the patient had an acute episode of TTP with superimposed severe preeclampsia. A cesarean delivery under general anesthesia was performed obtaining a 3 pound, 7 ounce infant. She received $2 \mathrm{U}$ of packed red blood cells and one pack of platelets. During her postpartum course, she underwent plasmapheresis and received methylprednisolone every other day for 1 week and received magnesium sulfate for 24 hours. Her platelets were $96,000 / \mu \mathrm{L}$ when she developed a febrile postpartum endometritis requiring parenteral antibiotics. On postoperative day 15 , the patient was discharged in stable condition with platelets in the 100,000 s.

\section{Discussion}

Laboratory values may not help in differentiating the two conditions because many of the characteristic laboratory features of both severe preeclampsia and TTP are similar. Furthermore, the usefulness of ADAMTS-13 serum activity level is limited in diagnosing TTP in pregnancy, as it appears to decrease as a physiological response to pregnancy and preeclampsia.

Treatment protocols for both conditions separately are standardized. Plasma exchange, even in pregnancy, is the current gold standard to prevent and treat autoantibodydriven ADAMTS-13-deficient TTP. Plasma exchange therapy has consistently reduced the mortality from 90 to $\sim 25 \%{ }^{2}$ Our patient did not immediately receive plasmapheresis because the decision to intervene with plasma exchange is based upon the severity of thrombocytopenia and microangiopathic hemolytic anemia and presence of neurological abnormalities and renal failure. Although there may be some anecdotal evidence ${ }^{5}$ that delivery is an optional therapy for an acute episode of TTP in pregnancy, it is not universally accepted.

There are newer approaches to treat TTP. Rituximab, a monoclonal antibody against mature B cells, is currently being used to treat TTP because treatments for TTP should be immunomodulatory. It acts to reduce immunoglobulin $G$ antibodies against ADAMTS $-13^{6}$ and may be one of the safest adjunct therapies for the continuation of pregnancy.

Our case also illustrates the importance of proper and timely use of hospital resources and of maintaining a designated service that is ultimately responsible for the coordination of care. Complex life-threatening conditions that are managed by a multidisciplinary team can result in unfocused and ineffective care that delays definitive management. Our patient benefitted not only from the clinical input of her consultants, but also from the structure of the care plan. The obstetric team remained the designated captain of the ship. The patient and her family were extensively counseled and notes of the content left on her chart to avoid any confusion that could occur when nurse or resident rounds were made later by team members who were not present during the initial family counseling.

\section{References}

1 Martin JN Jr, Bailey AP, Rehberg JF, Owens MT, Keiser SD, May WL. Thrombotic thrombocytopenic purpura in 166 pregnancies: 1955-2006. Am J Obstet Gynecol 2008;199:98-104

2 Crowther MA, George JN. Thrombotic thrombocytopenic purpura: 2008 update. Cleve Clin J Med 2008;75:369-375

3 Dashe JS, Ramin SM, Cunningham FG. The long-term consequences of thrombotic microangiopathy (thrombotic thrombocytopenic purpura and hemolytic uremic syndrome) in pregnancy. Obstet Gynecol 1998;91(5 Pt 1):662-668

4 Cunningham FG, Lindheimer MD. Hypertension in pregnancy. N Engl J Med 1992;326:927-932

5 Natelson EA, White D. Recurrent thrombotic thrombocytopenic purpura in early pregnancy: effect of uterine evacuation. Obstet Gynecol 1985;66(3):(, Suppl):54S-56S

6 George JN. Clinical practice. Thrombotic thrombocytopenic purpura. N Engl J Med 2006;354:1927-1935 